\title{
BIODIVERSITY RESOLUTION THROUGH SHE ANALYSIS
}

HAYEK, Lee-Ann C., NHB MRC-136, Smithsonian Institution, Washington, D.C. 20560, U.S.A.; BUZAS, Martin A., NHB MRC-121, Smithsonian Institution, Washington, D.C. 20560, U.S.A.

After 50 years of ecological research, the relationship of the number of individuals, $\mathrm{N}$, and species, $\mathrm{S}$, has never been integrated with population structure. The insurmountable problems have been: 1 . the correlation of quantitative measures and convergence of statistical distributions with sample size, and 2. the inability to separate any diversity statistic into species richness (S) and abundance components (E).

Here, instead of considering sample size dependency as a deficiency, we show that it is actually the missing requirement for identification of biodiversity patterns. We present a decomposition formula using the information function, $\mathrm{H}$, not as a diversity measure by itself, but as a vehicle for extricating orthogonal $\mathrm{S}$ and $\mathrm{E}$ components.

By examining patterns as well as values of $\mathrm{S}, \mathrm{H}$, and $\mathrm{E}$ with accumulating biological samples $(\mathrm{N})$, not only is the problem of convergence overcome so that the most commonly-used statistical distributions are distinguishable, but also a structured order is revealed undetectable by any other approach.

As an example, one hectare plots of trees in Bolivia and Guyana are shown to exhibit uniquely distinct and heretofore unseen patterns. 